\title{
Características de la colocación en voleibol, en etapas de formación, en sets ganados y perdidos Characteristics of the set in volleyball, in formative stages, in set win and lost Jara Gónzález-Silva, Alberto Moreno Domínguez, Carmen Fernández-Echeverría, Manuel Conejero Suarez, M. Perla Moreno Arroyo \\ Universidad de Extremadura(España)
}

\begin{abstract}
Resumen. El objetivo de la presente investigación fue conocer la asociación existente entre el resultado final del set y variables de la acción de colocación en el complejo I (KI) y complejo II (KII). La muestra del estudio estuvo formada por un total de 1209 acciones de colocación (856 en KI y 353 en KII), realizadas por los 16 equipos, participantes en el Campeonato de España de Selecciones Autonómicas de categoría cadete masculina. Las variables del estudio fueron el resultado del set y variables de la acción de colocación en KI y KII (zona de colocación, tipo de colocación, técnica de colocación, eficacia de la colocación, zona de envío de la colocación y tiempo de colocación). Los resultados de nuestro estudio mostraron que, en ambos complejos de juego, existía una asociación significativa entre el resultado del set y la zona de colocación, la técnica de colocación y la eficacia de colocación. En KI, los resultados mostraron que existía una asociación significativa entre el resultado del set y el tipo de colocación, la zona de envío de la colocación y el tiempo de colocación. Ninguna variable mostró asociación únicamente en KII. La colocación, a pesar de ser una acción de juego intermedia, mediante la cual no es posible obtener punto directamente, posee gran relevancia en el juego, asociándose determinados rasgos de la misma (en KI y KII) al resultado del set.
\end{abstract}

Palabras Clave. análisis de juego, colocación, masculino, cadete, complejos de juego.

Abstract. The aim of the study was to determine the relationship between the result of the set and variables of setting action in the complex I (KI) and complex II (KII). The study sample consisted of 1209 setting actions (856 in KI and 353 in KII), corresponding to the 16 male teams involved in the Spanish Championship in U-16 category. The study variables were result of set and setting variables in KI and KII (zone of set, type of set, setting technique, setting effectiveness, set area and time of setting). The results showed, in both game complexes, significant association between the result of the set and zone of set, setting technique, and setting effectiveness. In KI, the results showed a significant association between the result of set and type of set, the set area and time of setting. No variables displayed associations only in the KII. The setting action, despite being an intermediate game action, through which it is not possible to obtain direct points, has great relevance in the game, and some of its aspects are associated (in KI and KII) with the result of the set.

Keywords. game analysis, set, male, U-16, complex of game.

\section{Introducción}

En voleibol existen dos grandes complejos de juego, KI y KII. El complejo de ataque o KI es conocido como la fase de ataque. El objetivo de esta fase es la de neutralizar el saque del equipo contrario y, mediante un buen ataque (Papadimitriou, Pashali, Sermaki, Mellas \& Papas, 2004), conseguir el punto y la posesión del saque (Monteiro, Mesquita \& Marcelino, 2009). Las acciones que se producen en esta fase son la recepción del saque, la colocación y el ataque (Monteiro, et al., 2009).

El complejo de defensa o KII es conocido como la fase de defensa. El objetivo principal del KII es neutralizar y contrarrestar el ataque del equipo contrario, posibilitando una óptima construcción del contraataque, que permita la consecución del punto y la continuidad en la posesión del saque (Ureña, Calvo \& Lozano, 2002). Las acciones que se producen en el KII son el bloqueo, defensa en campo, colocación, contraataque y cobertura al contraataque (Palao, Santos \& Ureña, 2004).

Tanto en KI como en KII, la colocación es una acción de gran relevancia. El objetivo principal de la colocación es conseguir que los atacantes se encuentren ante las mejores condiciones posibles para realizar el ataque. Esta acción es fundamental para la organización ofensiva en ambos complejos de juego (Zetous, Moustakidis, Tsigilis \& Komnonakidou, 2007), pudiendo llegar a existir una relación de dependencia entre la colocación y el éxito del partido (Buscà \& Febrer, 2012).

La colocación es el segundo contacto y es realizada por un jugador especializado, el colocador. Este jugador es esencial en el voleibol (Matias \& Greco, 2011) debido a que es el encargado de la organización ofensiva del equipo (Zetou, et al., 2007). El colocador tiene que tener un amplio conocimiento táctico (Matias \& Greco, 2013) recayendo sobre él la decisión de distribución del ataque, es decir, de hacia dónde va el balón para realizar el ataque. Además de ello, de las habilidades de este jugador depende la velocidad y la diversidad del ataque (Mesquita \& Graça, 2002), llegando incluso a conseguir, en ocasiones, realizar óptimas colocaciones a partir de recepciones poco exitosas (Papadimitriou, et al.,

Fecha recepción: 10-15-15. Fecha de aceptación: 08-02-16 Jara Gónzález-Silva

gonzalezsilvajara@gmail.com
2004; Matias \& Greco, 2013).

El carácter interactivo del voleibol hace que las condiciones situacionales puedan tener efecto en el rendimiento (Taylor, Mellalieu, James \& Shearer, 2008). Esto ha producido que en la actualidad, surjan un gran número de investigaciones en las se estudia el rendimiento en las acciones de juego, considerando el contexto o situación de juego en las que se produce el mismo. Entre las variables situacionales consideradas en estos estudios podemos destacar la calidad del oponente, el resultado del partido, el resultado del set y la localización del partido (Campos, Stranganélli, Campos, Pasquarelli \& Gómez, 2014; Claver, Jiménez, Gil, Moreno \& Moreno, 2013; Marcelino, Sampaio \& Mesquita, 2012). Concretamente, el resultado del set, ha sido objeto de estudio en diferentes investigaciones, analizándose en ellas, la relación existente entre diferentes acciones de juego considerando esta variable situacional (Cox, 1984; Dávila \& García-Hermoso, 2012; Marelic, Resetar \& Jankovic, 2004).

Por todo ello, el objetivo de nuestro estudio es conocer la asociación existente entre el resultado final del set y variables de la acción de colocación, en ambos complejos de juego, en etapas de formación.

\section{Metodología}

\section{Participantes}

La muestra de estudio estuvo compuesta por un total de 1209 acciones de colocación (856 en KI y 353 en KII), realizadas por los 16 equipos, participantes en el Campeonato de España de Selecciones Autonómicas en categoría cadete masculina. Las colocaciones analizadas corresponden a la observación de un partido de cada uno de los equipos participantes en el campeonato.

\section{Variables}

Seguidamente presentamos las variables de estudio consideradas en nuestra investigación. Resultado del set, definido como la puntuación conseguida una vez finalizado el set. Las categorías consideradas en esta variable fueron set ganado y set perdido. Zona de colocación (figura 1), definida como el lugar del campo desde donde se realiza el pase de colocación. Las categorías consideradas en esta variable fueron zona 
excelente/zona A(área de $8 \mathrm{~m} 2,4$ metros de largo por 2 metros de ancho, ubicada a 2 metros de la línea lateral derecha y a 3 metros de la línea lateral izquierda), zona aceptable/ zona B (área de $6 \mathrm{~m} 2$, a 2 metros de la zona Ay 3 metros de ancho, ubicado a 2 metros de la línea lateral derecha y a 4 metros de la línea lateral izquierda), y zona no aceptable/zona C (que equivale a todo el área de juego excluidas las zonas Ay B), dichas categorías fueron también empleadas en el estudio de Castro \& Mesquita

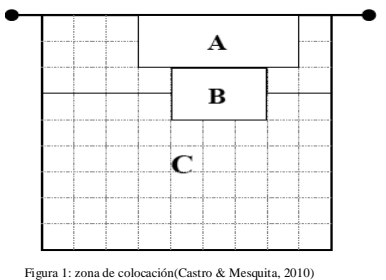

(2010).

Tipo de colocación, definida como la tipología de colocación que realiza el jugador, considerando si el colocador se encuentra en contacto con el suelo o no en el momento de realizar la colocación. Las categorías consideradas en esta variable fueron colocación en apoyo y colocación en salto (Palao \& Martínez, 2013; Palao \& Ahrabi-Fard, 2014). Eficacia de la colocación, hace referencia al rendimiento o efecto obtenido en la colocación. Se utilizaron los criterios del sistema FIVB, adaptado de Coleman (1975), agrupando algunos valores que aparecían diferenciados en dicho sistema: colocación mala, el pase de colocación no permite el ataque; colocación buena, el pase de colocación permite el ataque pero no con todas las opciones; y colocación perfecta, el pase de colocación permite el ataque con todas las opciones posibles (Palao \& Martínez, 2013). Zona hacia donde se envía la colocación, definido como la zona del campo donde se realiza el golpe de ataque. Las categorías consideradas en esta variable fueron zona zaguera, zona 2, zona 3 , y zona 4 (Papadimitriou, et al., 2004). Tiempo de colocación, definido como la interacción entre el momento en el que el colocador contacta con la pelota y el inicio de aproximación del atacante. Las categorías consideradas en esta variable fueron primer tiempo, segundo tiempo y tercer tiempo (Afonso, et al., 2010; Palao, Manzanares \& Ortega, 2009; Papadimitriou, et al., 2004).

\section{Procedimiento}

Mediante la observación sistemática se realizó la medida de las variables anteriormente indicadas. Las grabaciones de los partidos fueron realizadas con una cámara digital SONY HDR-XR155 sobre formato M2TS. Dicha cámara se ubicaba en uno de los fondos de la cancha de juego, garantizando una altura de 5 metros sobre el suelo, para obtener un óptimo plano de visión.

Una vez realizada la recogida de datos se procedió a la observación de todos los partidos por dos observadores. Para garantizar la fiabilidad de la observación, un observador, con experiencia en esta función y conocedor del voleibol, realizó un proceso de entrenamiento en el que se utilizaron, en las diferentes sesiones de entrenamiento, muestras con distintas características, y superando el $10 \%$ de la muestra total, indicado por Tabachnick \& Fidell (2007). Se alcanzaron en la observación de todas las variables unos valores de Kappa de Cohen inter-observador e intra-observador superiores a .75 (Fleiss, Levin, \& Paik, 2003).

\section{Análisis Estadístico}

En primer lugar, se realizó un análisis descriptivo con el objetivo de conocer las frecuencias de cada una de las variables estudiadas. En segundo lugar, se realizó un análisis inferencial para comprobar las asociaciones entre cada una de las variables estudiadas y el tipo de colocación. Este análisis se presenta a través de las tablas de contingencia incluyendo los valores de Chi-Cuadrado y V de Cramer. El nivel de significación estadística que se consideró fue de $\mathrm{p}<.05$.

\section{Resultados}

\section{Análisis descriptivo}

El análisis descriptivo en el complejo KI, muestra que el mayor número de colocaciones se realizan desde una zona excelente (41.1\%). Las características predominantes de la colocación son: la realización de colocaciones en apoyo (78.7\%), con una técnica de dedos (76.3\%) y con una eficacia perfecta(38.8\%). Además de ello, la zona predominante de destino de la colocación es la zona cuatro (46\%), y el tiempo de colocación más frecuente es el tercer tiempo (67.1\%).

En cuando al KII, los resultados muestran que la zona de colocación desde donde habitualmente se realiza la colocación es la zona excelente (42.2\%). En este complejo, el colocador realiza un mayor número de colocaciones en apoyo (75.1\%), colocaciones de dedos (79\%) y colocaciones con eficacia perfecta (38.8\%). La zona más habitual de destino de la colocación en es la zona cuatro (43.2\%), siendo las colocaciones a terceros tiempos las más frecuentes (70.2\%).

\section{Análisis inferencial}

\section{Análisis inferencia en KI.}

El análisis estadístico permite verificar la existencia de asociación significativa entre el resultado del set y la zona de colocación en KI, $\left(\mathrm{X}^{2}=27.371^{\mathrm{a}}\right.$; $\mathrm{V}$ de Cramer =.179; $\left.\mathrm{p}<.001\right)$. Las celdas que contribuyen de manera positiva a esta asociación son: la zona excelente con el set ganado, y la zona no aceptable con el set perdido (tabla 1).

El análisis estadístico permite verificar la existencia de asociación significativa entre el resultado del set y el tipo de colocación en KI, $\left(\mathrm{X}^{2}=14.632^{\mathrm{a}} ; \mathrm{V}\right.$ de $\left.\mathrm{Cramer}=.131 ; \mathrm{p}<.001\right)$. Las celdas que contribuyen de manera positiva a esta asociación son: la colocación en salto con el set ganado y la colocación en apoyo con el set perdido (tabla 2).

Tabla 1.

Tabla de contingencia resultado del set - zona de colocación en KI

\begin{tabular}{|c|c|c|c|c|c|}
\hline & & & \multicolumn{3}{|c|}{ Resultado del set } \\
\hline & & & Ganado & Perdido & Total \\
\hline \multirow{9}{*}{$\begin{array}{l}\text { Zona de } \\
\text { colocación } \\
\text { en KI }\end{array}$} & & Recuento & 91 & 151 & 242 \\
\hline & Zona Aceptable & Frecuencia esperada & 92.7 & 149.3 & 242.0 \\
\hline & & Residuos corregidos & -.3 & .3 & \\
\hline & & Recuento & 70 & 192 & 262 \\
\hline & Zona no aceptable & Frecuencia esperada & 100.4 & 161.6 & 262.0 \\
\hline & & Residuos corregidos & -4.6 & 4.6 & \\
\hline & & Recuento & 167 & 185 & 352 \\
\hline & Zona excelente & Frecuencia esperada & 134.9 & 217.1 & 352.0 \\
\hline & & Residuos corregidos & 4.6 & -4.6 & \\
\hline \multirow{2}{*}{ Total } & & Recuento & 328 & 528 & 856 \\
\hline & & Frecuencia esperada & 328.0 & 528.0 & 856.0 \\
\hline
\end{tabular}

$\underline{92.73}$

Tabla 2.

Tabla de contingencia resultado del set - tipo de colocación en KI

\begin{tabular}{|c|c|c|c|c|c|}
\hline & & & \multicolumn{3}{|c|}{$\begin{array}{l}\text { Resultado del set } \\
\end{array}$} \\
\hline & & & Ganado & Perdido & Total \\
\hline \multirow{6}{*}{$\begin{array}{l}\text { Tipo de } \\
\text { colocación en } \\
\text { KI }\end{array}$} & \multirow{3}{*}{ Colocación en salto } & Recuento & 92 & 90 & 182 \\
\hline & & Frecuencia esperada & 69.7 & 112.3 & 182.0 \\
\hline & & Residuos corregidos & 3.8 & -3.8 & \\
\hline & \multirow{3}{*}{ Colocación en apoyo } & Recuento & 236 & 438 & 674 \\
\hline & & Frecuencia esperada & 258.3 & 415.7 & 674.0 \\
\hline & & Residuos corregidos & -3.8 & 3.8 & \\
\hline \multirow{2}{*}{ Total } & & Recuento & 328 & 528 & 856 \\
\hline & & Frecuencia esperada & 328.0 & 528.0 & 856.0 \\
\hline
\end{tabular}

El análisis estadístico permite verificar la existencia de asociación significativa entre el resultado del set y la técnica de colocación en KI, $\left(\mathrm{X}^{2}=14.184^{\mathrm{a}} ; \mathrm{V}\right.$ de Cramer =.129; $\left.\mathrm{p}<.001\right)$. Las celdas que contribuyen de manera positiva a esta asociación son: la colocación de dedos con el set ganado y la colocación de antebrazos con el set perdido (tabla 3).

Tabla 3.

Tabla de contingencia resultado del set - técnica de colocación en KI

\begin{tabular}{|c|c|c|c|c|c|}
\hline & & & \multicolumn{3}{|c|}{ Resultado del set } \\
\hline \multirow{7}{*}{$\begin{array}{l}\text { Técnica de } \\
\text { colocación en KI }\end{array}$} & \multirow{4}{*}{$\begin{array}{l}\text { Colocación de } \\
\text { antebrazos }\end{array}$} & \multirow{4}{*}{$\begin{array}{l}\text { Recuento } \\
\text { Frecuencia esperada } \\
\text { Residuos corregidos }\end{array}$} & Ganado & Perdido & Total \\
\hline & & & 55 & 148 & 203 \\
\hline & & & 77.8 & 125.2 & 203.0 \\
\hline & & & -3.8 & 3.8 & \\
\hline & & Recuento & 273 & 380 & 653 \\
\hline & Colocación de dedos & Frecuencia esperada & 250.2 & 402.8 & 653.0 \\
\hline & & Residuos corregidos & 3.8 & -3.8 & \\
\hline & & Recuento & 328 & 528 & 856 \\
\hline & & Frecuencia esperada & 328.0 & 528.0 & 856.0 \\
\hline
\end{tabular}
77.79. 
El análisis estadístico permite verificar la existencia de asociación significativa entre el resultado del set y la eficacia de la colocación en KI, $\left(\mathrm{X}^{2}=31.090^{\mathrm{a}} ; \mathrm{V}\right.$ de $\left.\mathrm{Cramer}=.191 ; \mathrm{p}<.001\right)$. Las celdas que contribuyen de manera positiva a esta asociación son: la colocación perfecta con el set ganado y la colocación mala con el set perdido (tabla 4).

Tabla 4

Tabla de contingencia resultado del set - eficacia de la colocación en KI

\begin{tabular}{|c|c|c|c|c|c|}
\hline & & & \multicolumn{3}{|c|}{ Resultado del set } \\
\hline \multirow{10}{*}{$\begin{array}{l}\text { Eficacia de la } \\
\text { colocación en KI }\end{array}$} & \multirow{4}{*}{ Colocación mala } & \multirow[b]{2}{*}{ Recuento } & Ganado & Perdido & Total \\
\hline & & & 50 & 156 & 206 \\
\hline & & Frecuencia esperada & 78.9 & 127.1 & 206.0 \\
\hline & & Residuos corregidos & -4.8 & 4.8 & \\
\hline & & Recuento & 118 & 200 & 318 \\
\hline & Colocación buena & Frecuencia esperada & 121.9 & 196.1 & 318.0 \\
\hline & & Residuos corregidos & -.6 & .6 & \\
\hline & & Recuento & 160 & 172 & 332 \\
\hline & Colocación perfecta & Frecuencia esperada & 127.2 & 204.8 & 332.0 \\
\hline & & Residuos corregidos & 4.7 & -4.7 & \\
\hline Tht & & Recuento & 328 & 528 & 856 \\
\hline Total & & Frecuencia esperada & 328.0 & 528.0 & 856.0 \\
\hline
\end{tabular}

El análisis estadístico permite verificar la existencia de asociación significativa entre el resultado del set y lazona de envío de la colocación en $\mathrm{KI},\left(\mathrm{X}^{2}=17.534^{\mathrm{a}}\right.$; $\mathrm{V}$ de Cramer $=.143$; $\left.\mathrm{p}=.001\right)$. Las celdas que contribuyen de manera positiva a esta asociación son la zona 3 con el set ganado y la zona cuatro con el set perdido (tabla 5).

El análisis estadístico permite verificar la existencia de asociación significativa entre el resultado del set y el tiempo de colocación en KI, $\left(\mathrm{X}^{2}=29.533^{\mathrm{a}} ; \mathrm{V}\right.$ de Cramer $\left.=.186 ; \mathrm{p}<.001\right)$. Las celdas que contribuyen de manera positiva a esta asociación son: el primer y segundo tiempo con el set ganado y el tercer tiempo con el set perdido (tabla 6).

Tabla 5.

Tabla de contingencia resultado del set - zona de envío de la colocación en KI

\begin{tabular}{|c|c|c|c|c|c|}
\hline & & & \multicolumn{3}{|c|}{ Resultado del set } \\
\hline \multirow{13}{*}{$\begin{array}{l}\text { Zona de envío de la } \\
\text { colocación en KI }\end{array}$} & & \multirow[b]{2}{*}{ Recuento } & Ganado & Perdido & Total \\
\hline & \multirow{3}{*}{ Zona zaguera } & & 39 & 62 & 101 \\
\hline & & Frecuencia esperada & 38.7 & 62.3 & 101.0 \\
\hline & & Residuos corregidos & .1 & -.1 & \\
\hline & & Recuento & 82 & 144 & 226 \\
\hline & Zona dos & Frecuencia esperada & 86.7 & 139.3 & 226.0 \\
\hline & & Residuos corregidos & -.7 & .7 & \\
\hline & & Recuento & 73 & 62 & 135 \\
\hline & Zona tres & Frecuencia esperada & 51.8 & 83.2 & 135.0 \\
\hline & & Recuento & 4.1 & -4.1 & \\
\hline & & Frecuencia esperada & 134 & 259 & 393 \\
\hline & Zona cuatro & Residuos corregidos & 150.8 & 242.2 & 393.0 \\
\hline & & Recuento & -2.4 & 2.4 & \\
\hline \multirow{2}{*}{\multicolumn{2}{|c|}{ Total }} & Recuento & 328 & 527 & 855 \\
\hline & & Frecuencia esperada & 328.0 & 527.0 & 855.0 \\
\hline \multicolumn{6}{|l|}{ Tabla 6.} \\
\hline \multicolumn{6}{|c|}{ Tabla de contingencia resultado del set - tiempo de colocación en KI } \\
\hline & & & \multicolumn{3}{|c|}{ Resultado del set } \\
\hline \multirow{10}{*}{$\begin{array}{l}\text { Tiempo de } \\
\text { colocación en KI }\end{array}$} & \multirow{4}{*}{ Primer tiempo } & & Ganado & $\begin{array}{c}\text { Perdid } \\
\text { o }\end{array}$ & Total \\
\hline & & \multirow{3}{*}{$\begin{array}{l}\text { Recuento } \\
\text { Frecuencia esperada } \\
\text { Residuos corregidos }\end{array}$} & 57 & 38 & 95 \\
\hline & & & 36.4 & 58.6 & 95.0 \\
\hline & & & 4.6 & -4.6 & \\
\hline & \multirow{3}{*}{ Segundo tiempo } & Recuento & 83 & 103 & 186 \\
\hline & & Frecuencia esperada & 71.4 & 114.6 & 186.0 \\
\hline & & Residuos corregidos & 2.0 & -2.0 & \\
\hline & \multirow{3}{*}{ Tercer tiempos } & Recuento & 188 & 386 & 574 \\
\hline & & Frecuencia esperada & 220.2 & 353.8 & 574.0 \\
\hline & & Residuos corregidos & -4.8 & 4.8 & \\
\hline \multirow{2}{*}{ Total } & & Recuento & 328 & 527 & 855 \\
\hline & & Frecuencia esperada & 328.0 & 527.0 & 855.0 \\
\hline
\end{tabular}

\section{Análisis inferencia en KII.}

El análisis estadístico permite verificar la existencia de asociación significativa entre el resultado del set y la zona de colocación en KII, $\left(\mathrm{X}^{2}=19.048^{\mathrm{a}} ; \mathrm{V}\right.$ de Cramer=.232; $\left.\mathrm{p}<.001\right)$. Las celdas que contribuyen de manera positiva a esta asociación son: la zona excelente con el set ganado y la zona no aceptable con el set perdido (tabla 7)

El análisis estadístico no permite verificar la existencia de asociación significativa entre el resultado del set y el tipo de colocación en KII, $\left(\mathrm{X}^{2}=2.763^{\mathrm{a}}\right.$; V de Cramer=.088; $\left.\mathrm{p}=.096\right)$.
Tabla 7.

Tabla de contingencia resultado del set - zona de colocación en KII

\begin{tabular}{|c|c|c|c|c|c|}
\hline & & & \multicolumn{3}{|c|}{ Resultado del set } \\
\hline & & & Ganado $\mathbf{P}$ & Perdido & Total \\
\hline \multirow{9}{*}{$\begin{array}{l}\text { Zona de } \\
\text { colocación } \\
\text { en KII }\end{array}$} & \multirow{3}{*}{ Zona Aceptable } & Recuento & 72 & 30 & 102 \\
\hline & & Frecuencia esperada & 71.1 & 30.3 & 102.0 \\
\hline & & Residuos corregidos & .1 & -.1 & \\
\hline & \multirow{3}{*}{ Zona no aceptable } & Recuento & 56 & 46 & 102 \\
\hline & & Frecuencia esperada & 71.7 & 30.3 & 102.0 \\
\hline & & Residuos corregidos & -4.0 & 4.0 & \\
\hline & \multirow{3}{*}{ Zona excelente } & Recuento & 120 & 29 & 149 \\
\hline & & Frecuencia esperada & 104.7 & 44.3 & 149.0 \\
\hline & & Residuos corregidos & 3.6 & -3.6 & \\
\hline \multirow{2}{*}{ Total } & & Recuento & 248 & 105 & 353 \\
\hline & & Frecuencia esperada & 248.0 & 105 & 353.0 \\
\hline \multicolumn{6}{|c|}{0 casillas $(.0 \%)$ tienen frecuencia esperada inferior a 5 . La frecuencia mínima esperada es 30.34 . } \\
\hline \multicolumn{6}{|c|}{ Tabla 8.} \\
\hline Tabla de contin & ingencia resultado del set & t- técnica de colocación e & \multicolumn{3}{|c|}{ Resultado del set } \\
\hline \multirow{7}{*}{$\begin{array}{l}\text { Técnica de } \\
\text { colocación en } \\
\text { KII }\end{array}$} & \multirow{4}{*}{$\begin{array}{l}\text { Colocación de } \\
\text { antebrazos }\end{array}$} & & Ganado & o Perdido & Total \\
\hline & & Recuento & 39 & 35 & 74 \\
\hline & & Frecuencia esperada & 52.0 & 22.0 & 74.0 \\
\hline & & Residuos corregidos & -3.7 & 3.7 & \\
\hline & \multirow{3}{*}{ Colocación de dedos } & Recuento & 209 & 70 & 279 \\
\hline & & Frecuencia esperada & 196.0 & 83.0 & 279.0 \\
\hline & & Residuos corregidos & 3.7 & -3.7 & \\
\hline \multirow{2}{*}{ Total } & & Recuento & 248 & 105 & 353 \\
\hline & & Frecuencia esperada & 248.0 & 105.0 & 353.0 \\
\hline
\end{tabular}

0 casillas (.0\%) tienen frecuencia esperada inferior a 5. La frecuencia mínima esperada es 22.01

El análisis estadístico permite verificar la existencia de asociación significativa entre el resultado del set y la técnica de colocación en KII, $\left(\mathrm{X}^{2}=13.803^{\mathrm{a}} ; \mathrm{V}\right.$ de $\left.\mathrm{Cramer}=.198 ; \mathrm{p}<.001\right)$. Las celdas que contribuyen de manera positiva a esta asociación son: la colocación de dedos con el set ganado y la colocación de antebrazos con el set perdido (tabla 8).

El análisis estadístico permite verificar la existencia de asociación significativa entre el resultado del set y la eficacia de la colocación en $\mathrm{KII},\left(\mathrm{X}^{2}=15.017\right.$; ; de Cramer =.206; $\left.\mathrm{p}<.001\right)$. Las celdas que contribuyen de manera positiva a esta asociación son: la colocación perfecta con el set ganado y la colocación mala con el set perdido (tabla 9).

Tabla 9.

Tabla de contingencia resultado del set - eficacia de la colocación en KII

\begin{tabular}{|c|c|c|c|c|c|}
\hline & & & \multicolumn{3}{|c|}{ Resultado del set } \\
\hline \multirow{10}{*}{$\begin{array}{l}\text { Eficacia de la } \\
\text { colocación en } \\
\text { KII }\end{array}$} & & & Ganado & Perdido & Total \\
\hline & \multirow{3}{*}{ Colocación mala } & Recuento & 48 & 36 & 84 \\
\hline & & Frecuencia esperada & 59.0 & 25.0 & 84.0 \\
\hline & & Residuos corregidos & -3.0 & 3.0 & \\
\hline & \multirow{3}{*}{ Colocación buena } & Recuento & 89 & 43 & 132 \\
\hline & & Frecuencia esperada & 92.7 & 39.3 & 132.0 \\
\hline & & Residuos corregidos & -.9 & .9 & \\
\hline & \multirow{3}{*}{$\begin{array}{l}\text { Colocación } \\
\text { perfecta }\end{array}$} & Recuento & 111 & 26 & 137 \\
\hline & & Frecuencia esperada & 96.2 & 40.8 & 137.0 \\
\hline & & Residuos corregidos & 3.5 & -3.5 & \\
\hline \multirow{2}{*}{ Total } & & Recuento & 248 & 105 & 353 \\
\hline & & Frecuencia esperada & 248.0 & 105.0 & 353.0 \\
\hline
\end{tabular}

El análisis estadístico no permite verificar la existencia de asociación significativa entre el resultado del set y la zona de envío de la colocación en KII, ( $X^{2}=5.264^{\text {a; }}$ V de Cramer=.122; p=.153).

El análisis estadístico no permite verificar la existencia de asociación significativa entre el resultado del set y el tiempo de colocación en KII, $\left(\mathrm{X}^{2}=5.168^{\mathrm{a}}\right.$; V de Cramer =.121; $\left.\mathrm{p}=.075\right)$.

\section{Discusión}

El objetivo de nuestro estudio fue conocer la asociación existente entre el resultado final del set y variables de la acción de colocación, en ambos complejos de juego, en etapas de formación.

Las variables que mostraron asociación con el resultado del set tanto en el KI como en el KII fueron: zona de colocación, técnica de colocaciónyeficacia de colocación.

En la asociación entre la variable resultado del set y zona de colocación, nuestros resultados mostraron una asociación significativa, tanto en KI como en KII, entre ambas variables, de tal manera que era más 
frecuente de lo esperado por el azar, en ambos complejos de juego, que cuando se gana el set la colocación se realice desde una zona excelente y cuando se pierde el set, la colocación se realice desde una zona no aceptable.

Las investigaciones en análisis de juego en voleibol revelaron que existe una relación entre la calidad de la colocación y la zona de colocación (Afonso \& Mesquita, 2011). La relación entre estos dos aspectos justifica los resultados de nuestro estudio, donde se obtuvo que cuando el colocador realiza la colocación desde una zona excelente, incrementan las posibilidades de conseguir la victoria del set.

En la asociación entre la variable el resultado del set y la técnica de colocación, nuestros resultados mostraron una asociación significativa, tanto en KI como en KII, entre ambas variables, de tal manera que es más frecuente de lo esperado por el azar, en ambos complejos de juego, que cuando se gana el set la colocación se realice de dedos y cuando se pierde el set, la colocación se realice de antebrazos.

Palao, Manzanares \& Ortega (2009), en un estudio realizado en alto rendimiento, obtuvieron que la colocación de dedos era la técnica con la que se conseguía una mayor eficacia, pudiendo esto incrementar las posibilidades de éxito.

Entre las diferentes técnicas de ejecución de la colocación, la colocación de dedos es la técnica con la que se consigue una mayor precisión en la colocación (Ramos et al., 2004), pudiendo ser este uno de los motivos que justifican nuestros resultados.

En la asociación entre la variable el resultado del set y la eficacia de la colocación, nuestros resultados mostraron una asociación significativa, tanto en KI como en KII, entre ambas variables, de tal manera que es más frecuente de lo esperado por el azar, en ambos complejos de juego, que cuando se gana el set la colocación sea perfecta y cuando se pierde el set la colocación sea mala.

Silva, et al., (2013), exponían, en un estudio realizado en alto rendimiento, que podría existir una relación de dependencia entre la calidad de la colocación y el resultado final del partido, asociándose la colocación error con el fracaso (Silva, Lacerda \& Joa, 2014).

Un gran porcentaje de la eficacia del ataque se basa en la calidad de la acción de colocación (Bergeles, Barzouka \& Elissavet, 2009). Esto demuestra que la habilidad del colocador pueden influir en el éxito del equipo (Buscà \& Febrer, 2012), puesto que la realización de una colocación excelente hace que los equipos incrementen las posibilidades de ganar (Silva, et al., 2013).

Las variables que mostraron asociación con el resultado del set únicamente en KI y no en KII fueron: tipo de colocación, zona de envío de la colocación y tiempos de colocación.

En la asociación entre la variable resultado del set y tipo de colocación, nuestros resultados mostraron una asociación significativa, en KI, entre ambas variables, de tal manera que es más frecuente de lo esperado por el azar que cuando se gana el set la colocación se realice en salto y cuando se pierde el set, la colocación se realice en apoyo.

Palao \& Aharbi-Fard (2011), en un estudio en alto rendimiento, obtuvieron resultados que van en línea con los nuestros. Así, cuando las colocaciones se realizaban en salto había un incremento del éxito.

Con la colocación en salto el colocador consigue reducir el número de pistas que da al equipo rival y descender el tiempo de vuelo del balón (Palao \& Ahrabi-Fard, 2014), además de hacer que el balón llegue en condiciones óptimas a los atacantes, enfrentándose estos a un menor número de bloqueadores (Palao \& Martínez, 2013). Esto es debido a que la colocación en salto reduce las posibilidades de anticipación de los bloqueadores (Buscà \& Febrer, 2012). Todo ello hace que el uso de la colocación en salto incremente la eficacia de la acción de colocación (Palao \& Martínez, 2013) pudiendo ser este, uno de los motivos que justifiquen nuestros resultados.

En la asociación entre la variable resultado del set y zona de envío de la colocación, nuestros resultados mostraron una asociación significativa, en KI, entre ambas variables, de tal manera que es más frecuente de lo esperado por el azar que cuando se gana el set la colocación se envíe hacia zona tres y cuando se pierde el set, la colocación se envíe hacia zona cuatro.
En etapas de formación el colocador prefiere realizar colocaciones hacia zona cuatro, ya que, además de ser la zona donde encuentran los atacantes más fuertes y los bloqueadores rivales más débiles (Papadimitriou, et al., 2004), es la ejecución que los colocadores tienen más dominada, automatizada y la que se realiza con más seguridad (Costa, Mesquita, Greco, Ferreira \& Moraes, 2010).

A pesar de que los colocadores de categoría masculina muestran una mayor variabilidad que las de categorías femenina en distribución de la colocación (Mesquita \& Graça, 2002), las colocaciones hacia zona tres se realizan cuando las condiciones son ideales (Afonso, Mesquita, Marcelino \& da Silva, 2010). Además de ello, los ataques por zona cuatro disminuyen los ataques con los que se gana el punto (Palao \& Ahrabi-Fard, 2011), pudiendo ser este, uno de los motivos que justifiquen los resultados obtenidos en nuestra investigación.

En la asociación entre la variable el resultado del set y el tiempo de colocación, nuestros resultados mostraron una asociación significativa, en KI, entre ambas variables, de tal manera que es más frecuente de lo esperado por el azar que, cuando se gana el set la colocación se realice a primeros y segundos tiempos y cuando se pierde el set, la colocación se realice a terceros tiempos.

Patsiaouras, Kostantinos, Athanasios \& Kokaridas (2009), en un estudio realizado en alto rendimiento en categoría masculina, encontraron resultados similares a los nuestros obteniendo que los ataques rápidos eran predictores de la victoria.

El aumento del ritmo de juego puede favorecer el ataque (Zetou, et al., 2007), disminuyendo las posibilidades de continuidad del equipo rival cuando se realizan ataques rápidos (Castro, Souza \& Mesquita, 2011). Estos ataques producen un gran desequilibro en la defensa y crean un aumento en los errores en la defensa del equipo rival (Zetou, et al., 2007), incrementando esto, las posibilidades de conseguir el punto (Costa, et al., 2011) y de ganar (Costa, Afonso, Brant \& Mesquita, 2012).

Ninguna variable mostró asociación únicamente en KII y no en KI.

\section{Conclusiones}

En etapas de formación, en categoría masculina, en ambos complejos de juego, es más frecuente de lo esperado por el azar, ganar el set cuando los colocadores realicen colocaciones desde una zona excelente, con una técnica de dedos y con eficacia perfecta. El carácter cíclico del voleibol produce que cuando los balones llegan en buenas condiciones al colocador, esté pueda realizar la colocación en las mejores condiciones posibles, es decir, desde una zona excelente, de dedos, produciendo esto la realización de una colocación precisa.

En KI es más frecuente ganar el set cuando durante el mismo se realizan colocaciones en salto, hacia zona tres y a tiempos rápidos. La baja interferencia contextual del KI puede producir un incremento en la realización de colocaciones con características ideales que permiten incrementar la velocidad del juego en ataque. Por el contrario, en el KII, ninguna de estas tres variables (tipo de colocación, zona de envío de la colocación y tiempos de colocación) se ha asociado de forma significativa con el resultado del set, quizá debido al predominio del ataque sobre la defensa, y a la dificultad de conseguir condiciones óptimas para poder realizar un juego rápido de ataque en este complejo de juego.

En etapas de formación, en la categoría masculina, en función del nivel de desarrollo y dominio de los jugadores, sería conveniente valorar la posibilidad de incrementar la velocidad del juego en ataque, tanto en KI como en KII. Debido a que la colocación está influenciada por la acción anterior, se recomienda que, además de trabajar adecuadamente la colocación para que los jugadores adquieran un óptimo dominio técnico-táctico, se incida en el trabajo de las acciones previas (recepción y defensa).

\section{Referencias}

Afonso, J., \& Mesquita, I. (2011). Determinants of block cohesiveness and attack efficacy in high-level women's volleyball. European 
Journal of Sport Science, 11(1), 69-75.

Afonso, J., Mesquita, I., Marcelino, R., \& da Silva, A. (2010). Analysis of the setter's tactical action in high-performance women's volleyball. Kinesiology, 42(1), 82-89.

Asterios, P., Kostantinos, C., Athanasios, M., \& Dimitrios, K. (2009). Comparison of technical skills effectiveness of men's National Volleyball teams. International Journal of Performance Analysis of Sport, 9, 1-7.

Bergeles, N., Barzouka, K., \& Elissavet, N. (2009). Performance of male and female setters and attackers on Olympiclevel volleyball teams. International Journal of Performance Analysis in Sport, 9(1), 141-148.

Buscà, B., \& Febrer, J. (2012). Temporal fight between the middle blocker and the setter in high level volleyball. Revista Internacional de Medicina y Ciencias de la Actividad Física yel Deporte, 12(46), 313-327.

Campos, F., Stanganélli, L., Campos, L., Pasquearelli, B., \& Gómez, M. A. (2014). Performance indicators analysis at brazilian and italian women's volleyball leagues according to game location, game outcome, and set number. Perceptual \& Motor Skills, 118(2), 1-15.

Castro, J., \& Mesquita, I. (2010). Analysis of the attack tempo determinants in volleyball's complex II - a study on elite male teams. International Journal of Performance Analysis in Sport, 10(3), 197-206.

Castro, J. Souza, A., \& Mesquita, I. (2011).Attack efficacy in volleyball: elite male teams. Perfectual and Motor Skills, 113(2), 395-408.

Costa, G., Mesquita, I., Greco, P., Ferreria, N., \& Moraes, J. (2010). Relación entre el tipo, tiempo y el efecto del ataque en el voleibol femenino juvenil dealtonivel de competición. Motricidad.European Journal of Human Movement, 24, 121-132.

Costa, G., Caetano, C., Ferreira, N., Junqueira, G, Afonso, J., Costa, P., \& Mesquita, I. (2011). Determinants of attack tactics in Youth male elite volleyball. International Journal of Performance Analysis in Sport, 11, 96-104.

Costa, G, Afonso, J., Brant, E., \& Mesquita, I. (2012). Differences in game patterns between male and female youth volleyball. Kinesiology, 1, 60-66.

Claver, F., Jiménez, R., Gil, A., Moreno, A., \& Moreno, M.P. (2013). Relationship between performance in game actions and the match result. Astudy in volleyball training stages. Journal of Human and Exercise, 8(3), 651-659.

Dávila, C., \& García-Hermoso, A. (2012). El set cerrado en voleibol. Diferencias y poder discriminatorio de las acciones finales en etapas de formación. Retos. Nuevas tendencias en Educación Física, Deporte y Recreación, 21, 67-70.

Fleiss, J., Levin, B., \& Paik, M. (2003). Statistical methods for rates and proportions. NY: John Wiley \& Sons.

Marcelino, R., Sampaio, J., \& Mesquita, I. (2012). Attack and serve performances according to the match period and quality of opposition in elite volleyball matches. Journal of Strength and Conditioning Research, 26(12), 3385-3391.

Matias, C.J, \& Greco, P.J. (2011). Offensive organization assessment of winners of Brazilian Volleyball. Brazilian Journal of Physical Education and Sport, 33(4), 1007-1028.
Matias, C. J., \& Greco, P.J. (2013). O conhecimento tático declarativo dos levantadores campeoes de voleibol. Motriz, 19(1), 185-194.

Mesquita, I., \& Graça, A. (2002). Probing the strategic knowledge of an elite volleyball setter: Acase study. International Journal of Volleyball Research, 5(1), 13-17.

Monteiro, R., Mesquita, I., \& Marcelino, R. (2009). Relationship between the set outcome and the dig and attack efficacy in elite male, volleyball game. International Journal of Performance Analysis of Sport, 9. 294-305.

Palao, J., Santos, J., \& Ureña, A. (2004). Effect of team level on skill performance in volleyball. International Journal of Performance Analysis in Sport, 4(2), 50-60.

Palao, J. M., Manzanares, P., \& Ortega, E. (2009). Techniques used and efficacy of volleyball skills in relation to gender. International Journal of Performance Analysis of Sport, 9, 281-293.

Palao, J. M., \& Ahrabi-Fard, F. (2011). Side-out success in relation to setter's position on court in women's college volleyball. International Journal of Applied Sports Sciences, 23(1), 155-167.

Palao, J. M., \& Martínez, S. (2013). Utilización de la colocación en salto en función del nivel de competición en voleibol masculino. Revista Euroamericana de Ciencias del Deporte, 2(1), 43-49.

Palao, J. M., \& Ahrabi-Fard, I. (2014). Effect of jump set usage on sideout phase in women's college volleyball. Journal of Sport and Human Performance, 2(3), 1-10.

Papadimitriou, K., Pashali, E., Sermaki, I., Mellas, S., \& Papas, M. (2004). The effect of the opponents' serve on the offensive actions of Greek setters in volleyball games. International Journal of Performance Analysis in Sport, 4(1), 23-33.

Ramos, M. H., do Nascimiento, J. V., Donega, A. L., Novaes, A. J., de Souza, R., Silva, T. J., \& Lopes, A. (2004). Estructura interna das açoes de levantamento das equipes finalistas da superliga masculine de voleibol. Revista Brasileira de Ciencia e Movimento, 12(4), 3337.

Silva, M., Lacerda, D., \& João, P.V. (2013). Match analysis of discrimination skills according to the setter attack zone position in high level volleyball. International Journal of Performance Analysis in Sport, 13(2), 452-460.

Silva, M., Lacerda, D., \& Joao, P.V. (2014). Match analysis of discrimination skills according to the setter defence zone position in high level volleyball. International Journal of Performance Analysis in Sport, 14, 463-472.

Tabachnick, B. G. \& Fidell, L.S. (2007). Using multivariate statistics. 6th ed. Boston: Allyn and Bacon.

Taylor, J., Mellalieu, S., James, N., \& Shearer, D. (2008). The influence of match location, quality of opposition, and match status on technical performance in professional association football. Journal of Sports Sciences, 26, 885-895.

Ureña, A., Calvo, R.M., \& Lozano, C. (2002). Estudio de la recepción del saque en el voleibol masculino español de elite tras la incorporación del jugador líbero. Revista Internacional de Medicina y Ciencias de la Actividad Física y el Deporte, 2(4), 37-49.

Zetou, E., Moustakidis, A., Tsigilis, N., \& Komninakidou, A. (2007). Does Effectiveness of Skill in Complex I Predict Win in Men's Olympic Volleyball Games? Journal of Quantitative Analysis in Sports, 3(4), 1-9.

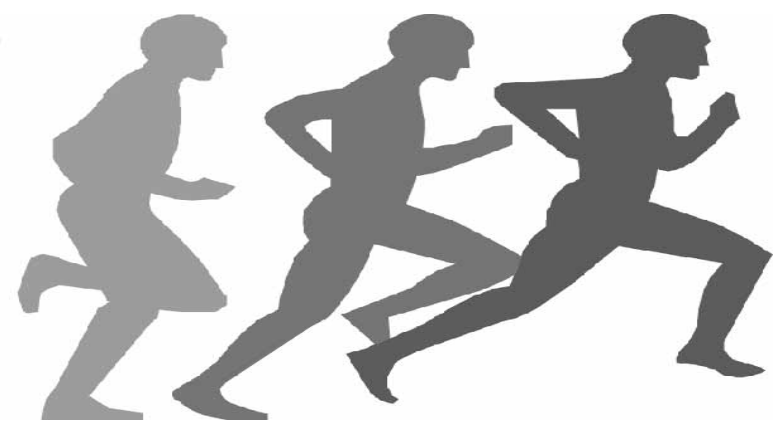

